\title{
Matrix oriented reduction of space-time Petrov-Galerkin variational problems.
}

\author{
Julian Henning, Davide Palitta, Valeria Simoncini, and Karsten Urban
}

\begin{abstract}
Variational formulations of time-dependent PDEs in space and time yield $(d+1)$-dimensional problems to be solved numerically. This increases the number of unknowns as well as the storage amount. On the other hand, this approach enables adaptivity in space and time as well as model reduction w.r.t. both type of variables. In this paper, we show that matrix oriented techniques can significantly reduce the computational timings for solving the arising linear systems outperforming both time-stepping schemes and other solvers.
\end{abstract}

\section{Introduction}

Time-stepping schemes based upon variational semi-discretizations are the standard approach for the numerical solution of time-dependent partial differential equations (PDEs). Using a variational formulation in space and a subsequent discretization e.g. in terms of finite elements, one is left with an evolution problem in time. Standard finite difference techniques then yield a time-marching scheme, where a spatial problem needs to be solved in each time step.

Even though theoretical investigations on space-time variational formulations of PDEs have been around for a long time, [10], it was seen prohibitive to treat the time

Julian Henning, Karsten Urban

Ulm University, Institute for Numerical Mathematics, Helmholtzstr. 20, 89081 Ulm (Ger-

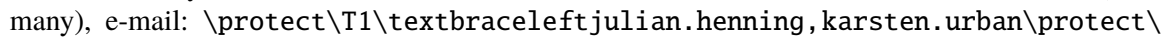
T1\textbraceright@uni-ulm.de

Davide Palitta

Max Planck Institute for Dynamics of Complex Technical Systems, Sandtorstr. 1, 39106 Magdeburg (Germany), e-mail:palitta@mpi-magdeburg.mpg.de

Valeria Simoncini

Università di Bologna, Centro AM², Dipartimento di Matematica, Piazza di Porta S. Donato 5, 40127 Bologna (Italy), and IMATI-CNR, Pavia, e-mail: valeria.simoncini@unibo.it 
as an additional variable for numerical simulations. In fact, if $\Omega \subset \mathbb{R}^{d}$ denotes the spatial domain, adding the time $t \in(0, T)=: I$ as an additional unknown results in a PDE on $\Omega_{I}:=I \times \Omega$ in Dimension $d+1$, which is costly both w.r.t. the amount of storage and the required computation time.

Also due to the increasing computing power the point of view has changed over the past years. In fact, being able to simulate problems for $d$ up to three until about 20 years ago, adding another dimension seemed to be impossible. Nowadays, where we face high-dimensional problems (e.g. from quantum physics or finance) with $d \gg 100$, adding another dimension seems almost negligible. Another aspect to use space-time variational problems arose from model reduction of parameterized time-dependent PDEs. In fact treating both time and space as variables allows one to perform model reduction for space and time, [14]. The time-stepping model reduction approach yields a time-marching scheme for a reduced spatial dimension but with the same number of time steps, [7].

In this paper, we address the question of how to efficiently solve the linear systems arising from a (full) Petrov-Galerkin discretization of space-time variational formulations of time-dependent PDEs. It turns out that the involved coefficient matrices, combining space and time discretizations, have a tensorproduct structure, which allows us to use more efficient matrix equations solvers than what can be done with the usual vector representation.

This paper is organized as follows: In $\$ 2$ we review space-time variational formulations of some PDEs and describe corresponding Petrov-Galerkin discretizations as well as the arising linear systems in $\$ 3, \$ 4$ is devoted to the description of the numerical schemes and $\$ 5$ to numerical experiments, in particular the comparison with time-stepping schemes.

\section{Space-Time Variational Formulation of PDEs}

The Heat Equation. Let $A: X \rightarrow X^{\prime}$ be an elliptic operator on $X:=H_{0}^{1}(\Omega)$ associated to a coercive bilinear form $a: X \times X \rightarrow \mathbb{R}$, and $f \in L_{2}\left(I ; X^{\prime}\right)$. We look for $u \in U:=H_{(0)}^{1}\left(I ; X^{\prime}\right) \cap L_{2}(I ; X)$ such that ${ }^{1} u_{t}+A u=f, u(0)=0$, where homogeneous initial conditions are chosen only for convenience. The variational formulation then reads

$$
\text { find } u \in U: \quad b(u, v)=\langle f, v\rangle \quad \text { for all } v \in V,
$$

where $V:=L_{2}(I ; X), b(u, v):=\int_{0}^{T} \int_{\Omega} u_{t}(t, x) v(t, x) d x d t+\int_{0}^{T} a(u(t), v(t)) d t$ and $\langle f, v\rangle:=\int_{0}^{T} \int_{\Omega} f(t, x) v(t, x) d x d t$. The well-posedness is ensured by Nečas' conditions, namely boundedness, injectivity and inf-sup condition of $b(\cdot, \cdot),[5]$.

The Wave Equation. Next, we consider an equation of wave type. Here, for $H:=$ $L_{2}(\Omega)$, we view the operator $A$ as a mapping $A: \operatorname{Dom}(A):=\{\phi \in H: A \phi \in$

${ }^{1} H_{(0)}^{1}\left(I ; X^{\prime}\right):=\left\{w: I \rightarrow X^{\prime}: w \in H^{1}\left(I ; X^{\prime}\right), w(0)=0\right\}$, recall that $H^{1}\left(I ; X^{\prime}\right) \hookrightarrow C\left(\bar{I} ; X^{\prime}\right)$. 
$H\} \rightarrow H$, or $A: H \rightarrow \operatorname{Dom}(A)^{\prime}$. For $f \in L_{2}(I ; H)$, we seek $u \in L_{2}(I ; H)$ such that $u_{t t}+A u=f, u(0)=0, u_{t}(0)=0$, where we choose homogeneous initial conditions again only for convenience. In this case, it is not so obvious how to setup a well-posed variational form. It turns out that a very-weak setting is appropriate. We formulate the problem as in (1) by using $U:=L_{2}(I ; H)$ as trial and $V:=\left\{v \in L_{2}(I ; H)\right.$ : $\left.v_{t t}+A v \in L_{2}(I ; H), v(T)=v_{t}(T)=0\right\}$ as test space. Then, one can show that (1) is well-posed for $b(u, v):=\left(u, v_{t t}+A v\right)_{L_{2}(I ; H)}$ and $\langle f, v\rangle:=(f, v)_{L_{2}(I ; H)}$ for fixed $T<\infty$.

\section{Petrov-Galerkin Discretizations}

In order to determine a numerical approximation to the solution of a variational problem (11), one chooses finite-dimensional trial and test spaces, $U_{\delta} \subset U, V_{\delta} \subset V$, respectively. For convenience, we assume that their dimension is equal, i.e., $N_{\delta}:=$ $\operatorname{dim} U_{\delta}=\operatorname{dim} V_{\delta}$. The Petrov-Galerkin method then reads

$$
\text { find } u_{\delta} \in U_{\delta}: \quad b\left(u_{\delta}, v_{\delta}\right)=\left\langle f, v_{\delta}\right\rangle \quad \text { for all } v_{\delta} \in V_{\delta} .
$$

As opposed to the coercive case, the well-posedness of (2) is not inherited from that of (11). In fact, the spaces $U_{\delta}$ and $V_{\delta}$ need to be appropriately chosen in the sense that the discrete inf-sup (or LBB -Ladyshenskaja-Babuška-Brezzi) condition holds, i.e., there exists an $\beta>0$ such that $\inf _{u_{\delta} \in U_{\delta}} \sup _{v_{\delta} \in V_{\delta}} \frac{b\left(u_{\delta}, v_{\delta}\right)}{\left\|u_{\delta}\right\|_{U}\left\|v_{\delta}\right\|_{V}} \geq \beta>0$, where the crucial point is that $\beta \neq \beta_{\delta}$. The size of $\beta$ is also crucial for the error analysis, since it holds that $\left\|u-u_{\delta}\right\|_{U} \leq \frac{1}{\beta} \inf _{w_{\delta} \in U_{\delta}}\left\|u-w_{\delta}\right\|_{U}$, [15].

The Heat Equation. Starting with the temporal discretization, choose some integer $N_{t}>1$ and set $\Delta t:=T / N_{t}$ resulting in a temporal triangulation $\mathcal{T}_{\Delta t}^{\text {time }} \equiv$ $\left\{t^{k-1} \equiv(k-1) \Delta t<t \leq k \Delta t \equiv t^{k}, 1 \leq k \leq N_{t}\right\}$ in time. Denote by $S_{\Delta t}=\operatorname{span}\left\{\sigma^{1}, \ldots, \sigma^{N_{t}}\right\}$ piecewise linear finite elements on $I$, where $\sigma^{k}$ is the (interpolatory) hat-function with the nodes $t^{k-1}, t^{k}$ and $t^{k+1}$ (resp. truncated for $\left.k \in\left\{0, N_{t}\right\}\right)$ and $Q_{\Delta t}=\operatorname{span}\left\{\tau^{1}, \ldots, \tau^{N_{t}}\right\}$ piecewise constant finite elements, where $\tau^{k}:=\chi_{I^{k}}$, the characteristic function on the temporal element $I^{k}:=\left(t^{k-1}, t^{k}\right)$. For the spatial discretization, we choose any conformal $X_{h}=\operatorname{span}\left\{\phi_{1}, \ldots, \phi_{N_{h}}\right\} \subset X$, e.g. piecewise linear finite elements. Then, we set $U_{\delta}:=S_{\Delta t} \otimes X_{h}, V_{\delta}=Q_{\Delta t} \otimes X_{h}$, $\delta=(\Delta t, h)$. It can be shown that this yields LBB. Moreover, for $A=-\Delta$ and choosing the energy norm on $X$ as well as a slightly modified norm on $U$, one can even prove that $\beta=1$, [14]. Finally, we remark that this specific discretization coincides with the Crank-Nicolson $(\mathrm{CN})$ scheme if a trapezoidal approximation of the righthand side temporal integration is used. Hence, we can later compare space-time Petrov-Galerkin numerical schemes with a $\mathrm{CN}$ time-stepping scheme.

Finally, we detail the linear system of equations $B_{\delta}^{T} u_{\delta}=f_{\delta}$, where 


$$
\begin{aligned}
{\left[B_{\delta}\right]_{(k, i),(\ell, j)} } & =\left(\dot{\sigma}^{k}, \tau^{\ell}\right)_{L_{2}(I)}\left(\phi_{i}, \phi_{j}\right)_{L_{2}(\Omega)}+\left(\sigma^{k}, \tau^{\ell}\right)_{L_{2}(I)} a\left(\phi_{i}, \phi_{j}\right), \\
{\left[f_{\delta}\right]_{(\ell, j)} } & =\left(f, \tau^{\ell} \otimes \phi_{j}\right)_{L_{2}(I ; H)},
\end{aligned}
$$

which means that we get a tensorproduct structure for the stiffness matrix $B_{\delta}=$ $D_{\Delta t} \otimes M_{h}+C_{\Delta t} \otimes A_{h}$, where the matrices are defined in an obvious manner. The right-hand side is not yet in a tensorproduct structure. However, we can achieve that by determining an approximation

$$
f(t, x) \approx \sum_{p=1}^{P} \vartheta_{p}(t) f_{p}(x)=: f^{P}(t, x),
$$

e.g. by the Empirical Interpolation Method (EIM), [2]. By choosing $P$ sufficiently large, we can achieve any desired accuracy. Then, we get $\left[f_{\delta}^{P}\right]_{(\ell, j)}=$ $\sum_{p=1}^{P}\left(\vartheta_{p}, \tau^{\ell}\right)_{L_{2}(I)}\left(f_{p}, \phi_{j}\right)_{L_{2}(\Omega)}$, i.e., $f_{\delta}^{P}=\sum_{p=1}^{P} h_{p} \otimes g_{p}$.

The Wave Equation. Constructing a stable pair of trial and test spaces for the wave equation is again a nontrivial task. Following an idea from [3], we first define the test space and construct the trial space in a second step in order to guarantee LBB, which, however, deteriorates with increasing $T$. Doing so, we set $R_{\Delta t}:=\operatorname{span}\left\{\varrho^{1}, \ldots, \varrho^{N_{t}}\right\} \subset H_{T}^{2}(I):=\left\{\rho \in H^{2}(I): \rho(T)=\dot{\rho}(T)=0\right\}$, e.g. piecewise quadratic splines on $\mathcal{T}_{\Delta t}^{\text {time }}$. For space, we choose any conformal $Z_{h}=\operatorname{span}\left\{\psi_{1}, \ldots, \psi_{N_{h}}\right\} \subset H^{2}(\Omega) \cap H_{0}^{1}(\Omega)$, e.g. piecewise quadratic finite elements. Then, we define $V_{\delta}:=R_{\Delta t} \otimes Z_{h}$, a tensor product space. The trial space $U_{\delta}$ is constructed by applying the adjoint PDE operator to each test basis function, i.e. $v_{k, i}:=\frac{d^{2}}{d t^{2}} \varrho^{k}(t) \psi_{i}(x)+A\left(\varrho^{k}(t) \psi_{i}(x)\right)=\varrho^{k}(t) \psi_{i}(x)+\varrho^{k}(t) A \psi_{i}(x)$. We detail the arising linear system of equations starting with the stiffness matrix

$$
\begin{aligned}
{\left[B_{\delta}\right]_{(k, i),(\ell, j)} } & =b\left(v_{k, i}, \varrho^{\ell} \otimes \psi_{j}\right)=\left(\ddot{\varrho}^{k} \otimes \psi_{i}+\varrho^{k} \otimes A \psi_{i}, \ddot{\varrho}^{\ell} \otimes \psi_{j}+\varrho^{\ell} \otimes A \psi_{j}\right)_{L_{2}(I ; H)} \\
& =\left(\ddot{\varrho}^{k}, \ddot{\varrho}^{\ell}\right)_{L_{2}(I)}\left(\psi_{i}, \psi_{j}\right)_{L_{2}(\Omega)}+\left(\varrho^{k}, \varrho^{\ell}\right)_{L_{2}(I)}\left(\psi_{i}, A \psi_{j}\right)_{L_{2}(\Omega)} \\
& +\left(\varrho^{k}, \ddot{\varrho}^{\ell}\right)_{L_{2}(I)}\left(A \psi_{i}, \psi_{j}\right)_{L_{2}(\Omega)}+\left(\varrho^{k}, \varrho^{\ell}\right)_{L_{2}(I)}\left(A \psi_{i}, A \psi_{j}\right)_{L_{2}(\Omega)}
\end{aligned}
$$

so that $B_{\delta}=Q_{\Delta t} \otimes M_{h}+\left(D_{\Delta t}+D_{\Delta t}^{T}\right) \otimes A_{h}+M_{\Delta t} \otimes Q_{h}$, again with obvious definitions of the matrices. For the right-hand side, we perform again an EIMtype approximation $f^{P}(t, x)$. Then, $\left[f_{\delta}^{P}\right]_{(\ell, j)}=\sum_{p=1}^{P}\left(\vartheta_{p} \otimes f_{p}, \varrho^{\ell} \otimes \psi_{j}\right)_{L_{2}(I ; H)}=$ $\sum_{p=1}^{P}\left(\vartheta_{p}, \varrho^{\ell}\right)_{L_{2}(I)}\left(f_{p}, \psi_{j}\right)_{L_{2}(\Omega)}$, so that the right-hand side has the same structure as in the first example. Due to the asymptotic behavior of the inf-sup-constant, we expect stability problems as $\Delta t \rightarrow 0$, i.e., $N_{t} \rightarrow \infty$.

\section{Efficient Numerical Methods for Tensorproduct Systems}

In both cases described above (and in fact also in space-time variational formulations of other PDEs), we obtain a (regular) linear system of the form 


$$
B_{\delta} u_{\delta}=f_{\delta} \quad \text { with } \quad B_{\delta}=\sum_{p=1}^{P_{B}} D_{p} \otimes A_{p}, \quad f_{\delta}=\sum_{\ell=1}^{P_{f}} h_{\ell} \otimes q_{\ell},
$$

where all involved matrices are sparse and (at least some of) the $A_{q}$ are s.p.d. Recall that $\left(D_{p} \otimes A_{p}\right) x=\operatorname{vec}\left(A_{p} X D_{p}^{T}\right)$, where vec stacks the columns of a given matrix one after the other, and $x=\operatorname{vec}(X)$. We can thus rewrite the system $B_{\delta} u_{\delta}=f_{\delta}$ in (6) as the linear matrix equation $\sum_{p=1}^{P_{B}} A_{p} U_{\delta} D_{p}^{T}=\sum_{\ell=1}^{P_{f}} q_{\ell} h_{\ell}^{T}$, with $u_{\delta}=\operatorname{vec}\left(U_{\delta}\right)$. Matrix equations are receiving significant attention in the PDE context, due to the possibility of maintaining the structural properties of the discretized problem, while limiting memory consumptions; see [13]. Under certain hypotheses, a large variety of discretization methodologies such as finite differences, isogeometric analysis, spectral (element) methods, certain finite element methods as well as various parametric numerical schemes rely on tensor product spaces; see, e.g., [1, 4, 8, 9]. More recently, all-at-once time discretizations have shown an additional setting where tensor product approximations naturally arise; see, e.g., [11] and references therein. Among the various computational strategies discussed in the literature [13], here we focus on projection methods that reduce the original equation to a similar one, but of much smaller dimension.

Discretized Heat Equation. The problem $B_{\delta} u_{\delta}=f_{\delta}$ stemming from 34 yields the following generalized Sylvester equation

$$
M_{h} U_{\delta} D_{\Delta t}+A_{h} U_{\delta} C_{\Delta t}=F_{\delta}, \quad \text { with } \quad F_{\delta}:=\left[g_{1}, \ldots, g_{P}\right]\left[h_{1}, \ldots, h_{P}\right]^{T} .
$$

The spatial stiffness and mass matrices $A_{h}$ and $M_{h}$ typically have significantly larger dimensions $N_{h}$ than the time discretization matrices $D_{\Delta t}, N_{\Delta t}$, i.e., $N_{t} \ll N_{h}$. We therefore use a reduction method only for the space variables by projecting the problem onto an appropriate space. A matrix Galerkin orthogonality condition is then applied to obtain the solution: given $V_{m} \in \mathbb{R}^{N_{h} \times k_{m}}, k_{m} \ll N_{h}$, with orthonormal columns, we consider the approximation space range $\left(V_{m}\right)$ and seek $Y_{m} \in \mathbb{R}^{k_{m} \times N_{t}}$ such that $U_{\delta, m}:=V_{m} Y_{m} \approx U_{\delta}$ and the residual $R_{m}:=F_{\delta}-\left(M_{h} U_{\delta, m} D_{\Delta t}+A_{h} U_{\delta, m} C_{\Delta t}\right)$ satisfies the Galerkin condition $R_{m} \perp \operatorname{range}\left(V_{m}\right)$. Imposing this orthogonality yields that $V_{m}^{T} R_{m}=0$ is equivalent to $V_{m}^{T} F_{\delta} V_{m}-\left(V_{m}^{T} M_{h} V_{m}\right) Y_{m} D_{\Delta t}-\left(V_{m}^{T} A_{h} V_{m}\right) Y_{m} C_{\Delta t}=0$. The resulting problem is again a generalized Sylvester equation, but of much smaller size, therefore Schur-decomposition oriented methods can cheaply be used, [13, sec.4.2], see [13] for a discussion on projection methods as well as their matrix and convergence properties.

For selecting $V_{m}$, let $F=F_{1} F_{2}^{T}$ with $F_{1}$ having full column rank. Given the properties of $A_{h}, M_{h}$, we propose to employ the rational Krylov subspace $\mathcal{R} \mathcal{K}_{m}:=$ $\operatorname{range}\left(\left[F_{1},\left(A_{h}-\sigma_{2} M_{h}\right)^{-1} M_{h} F_{1},\left(A_{h}-\sigma_{3} M_{h}\right)^{-1} M_{h} F_{1}, \ldots,\left(A_{h}-\sigma_{m} M_{h}\right)^{-1} M_{h} F_{1}\right]\right)$, where the shifts $\sigma_{s}$ can be determined adaptively while the space is being generated; see [13] for a description and references. The obtained spaces are nested, $\mathcal{R} \mathcal{K}_{m} \subseteq$ $\mathcal{R} \mathcal{K}_{m+1}$, therefore the space can be expanded if the approximation is not sufficiently good. To include a residual-based stopping criterion, the residual norm can be computed in a cheap manner, see, e.g., [6, 11] for the technical details.

Discretized Wave problem. The problem $B_{\delta} u_{\delta}=f_{\delta}$ now takes the matrix form 


$$
M_{h} U_{\delta} Q_{\Delta t}^{T}+A_{h} U_{\delta}\left(D_{\Delta t}+D_{\Delta t}^{T}\right)+Q_{h} U_{\delta} M_{\Delta t}=F_{\delta} .
$$

This three-term equation cannot be solved directly as before, therefore we opt for using preconditioned GMRES on the vectorized equation. The preconditioner is given by the functional $\mathcal{P}: U \rightarrow M_{h} U Q_{\Delta t}^{T}+Q_{h} U M_{\Delta t}$, corresponding to the discretized forth order operators, and exploits the matrix structure. Hence, at the $k$ th GMRES iteration we solve the generalized Sylvester equation $M_{h} W Q_{\Delta t}^{T}+Q_{h} W M_{\Delta t}=V_{k}$ where $V_{k}$ is such that $v_{k}=\operatorname{vec}\left(V_{k}\right)$ is the previous basis vector. Since in this one-dimensional problem dimensions are limited, this matrix equation is solved by explicitly diagonalizing the pairs $\left(Q_{h}, M_{h}\right)$ and $\left(Q_{\Delta t}, M_{\Delta t}\right)$ [13].

\section{Numerical Experiments}

In this section we show that the numerical solution of the linear system $B_{\delta} u_{\delta}=f_{\delta}$ can largely benefit from the exploitation of its Kronecker sum structure (6). The performance of the all-at-once methods is compared in terms of both computational time and memory requirements. For the heat equation, We also document the performances of $\mathrm{CN}$ in terms of computational time. We are not aware of any variant of $\mathrm{CN}$ that is able to exploit the low-rank structure of the underlying problem and we thus employ the classical $\mathrm{CN}$ scheme. Such implementation leads to large running times and a storage demand that is always equal to $N_{t} \cdot N_{h}$ as the full $U_{\delta}$ is allocated.

The tolerance of the final relative residual norm is set to $10^{-8}$ and in the following tables we also report the number of iterations needed to achieve such accuracy and the numerical rank of the computed solution. All results were obtained with Matlab R2017b on a machine with $2.1 \mathrm{GHz}$ processors and 192GB of RAM.

Example 5.1 (The heat equation) We consider the equation on the cube $\Omega=(-1,1)^{3}$ with homogeneous Dirichlet boundary conditions and the time interval $I:=(0,10)$ with initial conditions $u(0, x, y, z) \equiv 0$. The right-hand side is $f(t, x, y, z):=$ $10 \sin (t) t \cos \left(\frac{\pi}{2} x\right) \cos \left(\frac{\pi}{2} y\right) \cos \left(\frac{\pi}{2} z\right)$ and its discretized version is thus low rank. For discretization in space, linear finite elements were chosen, leading to the discretized generalized Sylvester equation in (7). We compare the performance of the Galerkin projection method based upon rational Krylov spaces described in $\$ 4$ (denoted RKSM) with that of a low-rank version of preconditioned GMRES (denoted LRFGMRES-RKSM). For the latter, the preconditioner is chosen as a fixed (five) number of iterations of the rational Krylov Galerkin projection method. Since the preconditioner is a non-linear operator, a flexible variant of GMRES is used. We refer the reader to [11] for more details. The results are displayed in Table 1

The $\mathrm{CN}$ method leads to an excessive workload compared with the all-at-once approaches for all considered values of $N_{h}$ and $N_{t}$, with the computational time growing linearly with the number of time steps $N_{t}$. The performance of the other methods is independent of the time discretization, and it only depends on the spatial component of the overall discrete operator. In fact, spatial mesh independence seems to also be achieved. 
Table 1: Results for Example 5.1 different values of $N_{h}$ and $N_{t}$. Memory allocations for RKSM and LRFGMRES+RKSM are given by $\mu_{\mathrm{mem}} \cdot\left(N_{h}+N_{t}\right)$.

\begin{tabular}{|rr|lrrr|rrrr|r|}
\hline & & \multicolumn{4}{|c|}{ RKSM } & \multicolumn{4}{|c|}{ LR-FGMRES+RKSM } & CN \\
$N_{h}$ & $N_{t}$ & Its $\mu_{\text {mem }}$ & $\operatorname{rank}\left(U_{\delta}\right)$ & Time $(\mathrm{s})$ & Its & $\mu_{\text {mem }}$ & $\operatorname{rank}\left(U_{\delta}\right)$ & Time $(s)$ & Time (s) \\
\hline 41300 & 100 & 16 & 17 & 10 & 88.74 & 4 & 83 & 13 & 143.13 & 296.16 \\
& 300 & 13 & 14 & 9 & 68.86 & 4 & 74 & 10 & 130.27 & 871.38 \\
& 500 & 13 & 14 & 9 & 65.88 & 4 & 75 & 11 & 134.73 & 1468.40 \\
\hline 347361 & 100 & 16 & 17 & 9 & 3144.02 & 4 & 71 & 10 & 4793.63 & 13805.09 \\
& 300 & 14 & 15 & 9 & 2673.83 & 4 & 78 & 9 & 4780.46 & 41701.10 \\
& 500 & 14 & 15 & 9 & 2699.98 & 4 & 80 & 9 & 4827.13 & 70044.52 \\
\hline
\end{tabular}

Example 5.2 (The wave equation) We consider the wave problem with $A=-\Delta$ on $\Omega=(0,1)$ with homogeneous Dirichlet boundary conditions and $I:=(0,1)$. Setting $f(t, x):=\sin (2 \pi x)+4 \pi^{2} t^{2} \sin (2 \pi x)$ yields the analytical solution $u(t, x)=$ $t^{2} \sin (2 \pi x)$. We choose cubic B-Splines for the discretization in space and time. The discretized problem thus leads to the matrix equation in (8). In Figure 1 we report some our preliminary results. Note, that our above discretization does not yield an equivalent time-stepping scheme with which we could do comparisons.

Fig. 1: Example 5.2 Left: Results for different values of $N_{h}$ and $N_{t}$. Right: Relative residual norm history for some values of $N_{h}$ and $N_{t}$.

\begin{tabular}{|rr|ccr|r|}
\hline & & \multicolumn{2}{|c|}{ GMRES+LYAP } & backslash \\
$N_{h}$ & $N_{t}$ & Its.rank $\left(U_{\delta}\right)$ Time $(\mathrm{s})$ & Time $(\mathrm{s})$ \\
\hline 256 & 256 & 16 & 13 & 0.21 & 1.17 \\
& 512 & 36 & 35 & 1.51 & 2.39 \\
1024 & 81 & 74 & 20.97 & 12.61 \\
\hline 512 & 256 & 26 & 31 & 0.61 & 2.30 \\
512 & 40 & 43 & 2.64 & 5.09 \\
1024 & 81 & 74 & 20.97 & 12.61 \\
\hline 1024 & 256 & 50 & 59 & 3.55 & 4.82 \\
512 & 68 & 72 & 10.12 & 11.13 \\
1024 & 102 & 92 & 54.15 & 24.28 \\
\hline
\end{tabular}

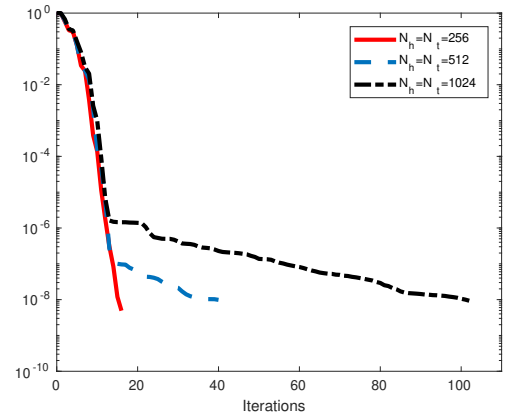

The table on the left shows that the performances of our preconditioned scheme are quite good for small values of $N_{h}$ and $N_{t}$. Indeed, in this case, the preconditioner manages to drastically reduce the number of iterations needed to converge so that GMRES+LYAP turns out to be faster than the Matlab solver backslash applied to the solution of the linear system $B_{\delta} u_{\delta}=f_{\delta}$, in spite of the 1D nature (in space) of the problem. However, the effectiveness of the adopted preconditioner worsens by increasing the number of degrees of freedom. This is due to a dramatic increment in the condition number of the coefficient matrices (see the discussion at the end of $\$ 3$ ) that causes an abrupt very slow decrement (almost stagnation) in the GMRES residual at the level that seems to be related to the conditioning of the involved matrices, see Fig. 11 (right). As it is, the problem associated with handling this ill-conditioning in the algebraic equation is crucial for the overall solver performance, and will be the topic of future works. Alternatively, one may try to directly address the solution of the multiterm matrix equation (8) as it is done in [12] for certain stochastic PDEs. 
Projection-based solvers may be very well-suited for our framework as the solution $U_{\delta}$ turns out to be low-rank. However, such an approach needs the design of ad hoc approximation spaces and we plan to pursue this challenging research direction in the near future.

\section{References}

1. M. Bachmayr, A. Cohen, and W. Dahmen. Parametric PDEs: sparse or low-rank approximations? IMA J. Numer. Anal., 38:1661-1708, 2018.

2. M. Barrault, Y. Maday, N. C. Nguyen, and A. T. Patera. An 'empirical interpolation' method: application to efficient reduced-basis discretization of partial differential equations. C. R. Math. Acad. Sci. Paris, 339(9):667-672, 2004.

3. J. Brunken, K. Smetana, and K. Urban. (Parametrized) First Order Transport Equations: Realization of Optimally Stable Petrov-Galerkin Methods. SIAM J. Sci. Comput., 41(1):A592A621, 2019.

4. W. Dahmen, R. DeVore, L. Grasedyck, and E. Süli. Tensor-sparsity of solutions to highdimensional elliptic partial differential equations. Found. Comput. Math., 16:813-874, 2016.

5. R. Dautray and J.-L. Lions. Mathematical analysis and numerical methods for science and technology. Vol. 5. Springer-Verlag, Berlin, 1992. Evolution problems. I.

6. V. Druskin and V. Simoncini. Adaptive rational Krylov subspaces for large-scale dynamical systems. Systems and Control Letters, 60:546-560, 2011.

7. S. Glas, A. Mayerhofer, and K. Urban. Two ways to treat time in reduced basis methods. In Model reduction of parametrized systems, volume 17 of MS\&A. Model. Simul. Appl., pages 1-16. Springer, Cham, 2017.

8. M. Griebel and H. Harbrecht. On the construction of sparse tensor product spaces. Math. Comp., 82(282):975-994, 2013.

9. B. N. Khoromskij and C. Schwab. Tensor-structured Galerkin approximation of parametric and stochastic elliptic PDEs. SIAM J. Scient. Comput., 33(1):364-385, 2011.

10. J.-L. Lions and E. Magenes. Problèmes aux limites non homogènes et applications. Vol. 2. Travaux et Recherches Mathématiques, No. 18. Dunod, Paris, 1968.

11. D. Palitta. Matrix equation techniques for certain evolutionary partial differential equations. arXiv math.NA, no. 1908.11851, 2019.

12. C. E. Powell, D. Silvester, and V. Simoncini. An efficient reduced basis solver for stochastic Galerkin matrix equations. SIAM J. Sci. Comput., 39(1):A141-A163, 2017.

13. V. Simoncini. Computational methods for linear matrix equations. SIAM Review, 58(3):377$441,2016$.

14. K. Urban and A. T. Patera. An improved error bound for reduced basis approximation of linear parabolic problems. Math. Comp., 83(288):1599-1615, 2014.

15. J. Xu and L. Zikatanov. Some observations on Babuška and Brezzi theories. Numer. Math., 94(1):195-202, 2003. 